\title{
Adenomyosis invades bladder-A case report
}

\author{
Tran Huy Dung ${ }^{1}$, Nguyen Thi Yen Thu ${ }^{1}$, Cao Van Hung ${ }^{1}$, Truong Thi Thuy Duong ${ }^{1}$, Pham Cong Toan ${ }^{1}$, Ho Minh Tuan ${ }^{1}$, Vu Tran Bao \\ $\mathrm{Chau}^{2}$ and Tran Thi Loi ${ }^{1 *}$
}

${ }^{1} \mathrm{OB} / \mathrm{GYN}$ Department, School of Medicine, Vietnam National University HCMc, Vietnam

${ }^{2}$ Faculty of Odonto-Stomatology, University of Medicine and Pharmacy HCMc, Vietnam

\begin{abstract}
Bladder endometriosis secondary to an adenomyosis is a rare variety of deep endometriosis with nonspecific symptoms such as dysuria, frequency, urgency, hematuria and bladder pain. We report a case of a 38-year-old woman with gravida 1, parity 1 presented to the department of urology at a polyclinic hospital for dysuria during her menstruation. Due to the diagnosis of bladder endometriosis she was treated with laparoscopic partial cystectomy and ureteral reimplantation and GnRH agonist $3.75 \mathrm{mg}$ each 4 weeks. After 4 months of $\mathrm{GnRH}$ agonist regimen, the patient suffered from severe menopausal disorders so she consulted with a gynecologist. The pelvic examination, ultrasound scan, and MRI (Magnetic Resonance Imaging) revealed an adenomyosis, a progestogen releasing intrauterine device (LNGIUD) was inserted. Management of bladder endometriosis may be required both surgical and medical treatment, so the patient should be treated at a referral hospital where cooperation between urologist and gynecologist is available.
\end{abstract}

\section{Introduction}

Endometriosis is a common and non-malignant disorder, known as the presence of endometrial glands and stroma developing outside the uterine cavity, then induces a chronic inflammatory reaction. The most frequent location of endometriosis is ovaries (endometrioma), sometimes it can be found in pelvic peritoneum including anterior and posterior pouch, myometrium (adenomyosis), rarely deep infiltrating endometriosis in recto-vaginal septum, urinary tract, pericardium and pleura... Endometriosis is a hormone-dependent disease that occurs from $10-15 \%$ in reproductive-age women who suffer chronic pelvic pain but up to $50 \%$ in infertile women with normal ovulation [1-3].

Pathogenesis of endometriosis can be explained by three theories including ectopic transplantation of endometrial tissue by means of retrograde menstruation, coelomic metaplasia and endometriosis may develop from rudimentary cells of Mullerian origin after prolonged hormonal stimulation. However, definitive cause of endometriosis remains unknown. Patient with endometriosis can have variable and nonspecific symptoms such as pelvic pain, dysmenorrhea, dyspareunia, ovarian mass or infertility. Interestingly, the severity of symptoms does not strongly correlate with the size and amount of endometriosis lesions so that endometriosis is definitively diagnosed by laparoscopy or histopathology of tissue biopsy.

Deeply infiltrating endometriosis is a very severe type of endometriosis which locates in sigma colon, rectovaginal septum, pouch of Douglas, and urinary tract (ureter or bladder). Urinary tract endometriosis involvement can occur in $1 \%$ patient with endometriosis, but up to $52 \%$ of deep endometriosis [4], especially bladder endometriosis has the most common frequency with 70\% [5]. Ectopic endometrium in the bladder can expand from the original adenomyosis, which causes bladder endometriosis. Symptoms of endometriosis in the bladder are usually nonspecific such as dysuria, heightened frequency, urgency, hematuria and bladder pain [4]. Because this disorder depends on estrogen, symptoms usually disappear after menopause. Diagnosis and treatment of bladder endometriosis or adenomyosis remain a challenge,so gynecologists and urologists should collaborate to solve it. Here, we report a case of bladder endometriosis due to adenomyosis invasion.

\section{Case report}

A 38-year-old woman with gravida 1, parity 1 (a 7-year-old girl) presented to the department of urology at a Polyclinic hospital for dysuria during her menstruation since 3 months ago. She suffered dysmenorrhea since her adolescence until now, but it was not severe so she didn't seek the gynecologic exammination . Physical examination and ultrasound scan indicated a suspicion of a bladder tumor. The patient went to a hospital in Singapore for cystoscopy, biopsy and histopathology. The report showed an endometriosis in bladder cavity. She returned to Vietnam due to unaffordability for treatment in Singapore.

In Vietnam, she came back to the department of urology. A laparoscopic partial cystectomy and ureteral re-implantation were performed by an urologist expert. The histopathology confirmed an endometriosis invading the bladder. The symptoms ameliorated only 1 month post-operation. Since the $2^{\text {nd }}$ month post-operation, the patient could not bear the frequent urination and dysuria. She was injected with GnRH agonist $3.75 \mathrm{mg}$ each 4 weeks. After 4 months of GnRH agonist regimen, the patient suffered from severe menopausal disorders: hot flush, vaginal atrophy,... Then, she consulted with a gynecologist. The pelvic examination, ultrasound scan, and MRI (Magnetic Resonance Imaging) revealed an adenomyosis, the uterus is equal an 8 weeks pregnancy. A progestogen releasing intrauterine device (LNGIUD)

Correspondence to: Tran Thi Loi, Professor, MD. PhD. Head of OB/GYN Department, School of Medicine, Vietnam National University HCMc, Vietnam, E-mail: tranthiloi@hotmail.com

Key words: deep endometriosis, adenomyosis, bladder endometriosis

Received: November 06, 2017; Accepted: November 27, 2017; Published: November 29, 2017 
was inserted. Six months after the insertion of LNG IUD, the patient had no dysmenorrhea, dysuria, and no frequent urination. She had no menstruation, but just spotting.

\section{Discussion}

Treatment of adenomyosis personalizes and depends on the severity of clinical symptoms such as dysmenorrhea and menorrhagia with the main purpose of treatment is to reduce pain and infertility. According to The European Society for Human Reproduction and Embryology (ESHRE) guidelines: management of women with painful endometriosis: these medications are recommended:[6]

- Progestagens [medroxyprogesterone acetate (oral or depot), dienogest, cyproterone acetate, norethisterone acetate or danazol] or anti-progestagens (gestrinone)(Level A)

- GnRH agonists (Level A)

- Levonorgestrel-releasing intrauterine system (LNG-IUS) (Level A)

- Hormonal contraceptives (Level B)

- Vaginal contraceptive ring or a transdermal (oestrogen/progestin) patch (Level C)

- NSAIDs is effective on primary dysmenorrhoea and are commonly used as a treatment of endometriosis-associated pain.

In a randomized clinical trial included 62 patients, comparing LNG - IUD and the low - dose combined oral contraceptions (COCs), the authors had conclusion that both may be resolved the pain and bleeding symptoms, however LNG - IUD has more effective than COCs [7].

About extragenital endometriosis: The evidence of treatment of extragenital endometriosis is limited and mainly published as case report so it's level D recommendation: surgical removal of symptomatic extragenital endometriosis, when possible, to relieve symptoms. When surgical treatment is difficult or impossible, medical treatment of extragenital endometriosis to relieve symptoms is considered.

This case is a rare condition that endometrial glands infiltrate deeply through myometrium to detrusor muscle of bladder. The treatment of bladder endometriosis are controversial and individual because the treatment depends on multifactor such as patient age, expecting of future pregnancy, coexisting pelvic diseases, fertility preferences, severity of lower urinary tract symptoms and degree of menstrual dysfunction. Treatment of bladder endometriosis can be medical or surgical, or combination. Medical therapy is the best temporary decision for treating symptomatic bladder endometriosis to relief pain and preserve fertility in young women wishing to conceive. Surgical treatment can be chosen for women whose medical therapy failed, or cannot receive medical. Depends on the location of endometriosis lesions with ureteral meatuses, a simple partial cystectomy or partial cystectomy with ureteral cannulation or ureteral reimplantation is performed with the aim to complete excision of the lesion. Partial cystectomy is a rather safe procedure because the bladder contents are sterilized, bladder has rich vascularization, and prolonged urinary drainage. Laparoscopy is preferred than laparotomy because the postoperative recovery time of laparoscopy is better. Endometriosis can be recurrent after surgery so that hormonal therapy should be nextstep treatment to reduce pain and avoid relapse $[8,9]$.

\section{Conclusion}

Endometriosis of bladder especial secondary to an adenomyosis is a complicated form of deep endometriosis. Management of bladder endometriosis may be required both surgical and medical treatment, so the patient should be treated at a referral hospital where cooperation between urologist and gynecologist is available.

\section{References}

1. Meuleman C, Vandenabeele B, Fieuws S, Spiessens C, Timmerman D, et al. (2009) High prevalence of endometriosis in infertile women with normal ovulation and normospermic partners. Fertil Steril 92: 68-74.

2. Mowers EL, Lim CS, Skinner B, Mahnert N, Kamdar N, et al. (2016) Prevalence of Endometriosis During Abdominal or Laparoscopic Hysterectomy for Chronic Pelvic Pain. Obstet Gynecol 127: 1045-1053. [Crossref]

3. Viganò P, Parazzini F, Somigliana E, Vercellini P (2004) Endometriosis: epidemiology and aetiological factors. Best Pract Res Clin Obstet Gynaecol 18: 177-200. [Crossref]

4. Knabben L, Imboden S, Fellmann B, Nirgianakis K, Kuhn A, et al. (2015) Urinary tract endometriosis in patients with deep infiltrating endometriosis: prevalence, symptoms, management, and proposal for a new clinical classification. Fertil Steril 103: 147-152.

5. Gabriel B, Nassif J, Trompoukis P, Barata S, Wattiez A (2011) Prevalence and management of urinary tract endometriosis: a clinical case series. Urology 78: 12691274. [Crossref]

6. Dunselman GA, Vermeulen N, Becker C, Calhaz-Jorge C, D'Hooghe T, et al. (2014) ESHRE guideline: management of women with endometriosis. Hum Reprod 29: 400412. [Crossref]

7. Shaaban OM, Ali MK, Sabra AM, Abd El Aal DE (2015) Levonorgestrel-releasing intrauterine system versus a low-dose combined oral contraceptive for treatment of adenomyotic uteri: a randomized clinical trial. Contraception 92: 301-307. [Crossref]

8. Kim KA, Yoon SW, Lee C, Seong SJ, Yoon BS, et al, Short-term results of magnetic resonance imaging-guided focused ultrasound surgery for patients with adenomyosis: symptomatic relief and pain reduction. Fertil Steril 95: 1152-1155. [Crossref]

9. Kitamura Y, Allison SJ, Jha RC, Spies JB, Flick PA, et al. (2006) MRI of adenomyosis changes with uterine artery embolization. AJR Am J Roentgenol 186: 855-864. [Crossref]

Copyright: (C2017 Dung TH. This is an open-access article distributed under the terms of the Creative Commons Attribution License, which permits unrestricted use, distribution, and reproduction in any medium, provided the original author and source are credited. 